\title{
Evaluation of a Novel Rapid Test System for the Detection of Specific IgE to Hymenoptera Venoms
}

\author{
Nikolai Pfender, ${ }^{1}$ Ralf Lucassen, ${ }^{2}$ Nadine Offermann, ${ }^{2}$ Johannes Schulte-Pelkum, ${ }^{2}$ \\ Margrit Fooke, ${ }^{2}$ and Thilo Jakob ${ }^{1}$ \\ ${ }^{1}$ Allergy Research Group, Department of Dermatology, University Medical Center Freiburg, Hauptstraße 7, 79104 Freiburg, Germany \\ ${ }^{2}$ Dr. Fooke Laboratorien GmbH, Habichtweg 16, 41468 Neuss, Germany
}

Correspondence should be addressed to Nikolai Pfender, nikolai.pfender@spitalaffoltern.ch

Received 15 September 2011; Revised 25 November 2011; Accepted 26 November 2011

Academic Editor: Ting Fan Leung

Copyright (C) 2012 Nikolai Pfender et al. This is an open access article distributed under the Creative Commons Attribution License, which permits unrestricted use, distribution, and reproduction in any medium, provided the original work is properly cited.

\begin{abstract}
Background. The Allergy Lateral Flow Assay (ALFA) is a novel rapid assay for the detection of sIgE to allergens. The objective of this study is the evaluation of ALFA for the detection of sIgE to bee venom (BV) and wasp venom (WV) in insect venom allergic patients. Methods. Specific IgE to BV and WV was analyzed by ALFA, ALLERG-O-LIQ, and ImmunoCAP in 80 insect venom allergic patients and 60 control sera. Sensitivity and specificity of ALFA and correlation of ALFA and ImmunoCAP results were calculated. Results. The sensitivity/specificity of ALFA to the diagnosis was 100\%/83\% for BV and $82 \% / 97 \%$ for WV. For insect venom allergic patients, the Spearman correlation coefficient for ALFA versus ImmunoCAP was 0.79 for BV and 0.80 for WV. However, significant differences in the negative control groups were observed. Conclusion. ALFA represents a simple, robust, and reliable tool for the rapid detection of sIgE to insect venoms.
\end{abstract}

\section{Introduction}

Reliable diagnosis of insect venom allergy is based on the combination of patient's history, skin testing (SPT) and laboratory tests for the detection of specific $\operatorname{IgE}$ (sIgE) [1-4].

The methodology for the measurement of sIgE has evolved during the recent years [5, 6]. Lately, rapid assays for the detection of sIgE as point-of-care diagnostics have been developed using various strategies $[7,8]$. ALFA, the Allergy Lateral Flow Assay (Dr. Fooke Laboratorien GmbH, Neuss, Germany), combines the advantages of lateral flow devices with the flexibility of choosing different allergens as already shown for cat epithelia (e1), Dermatophagoides pteronyssinus (d1), Alternaria alternata (m6), birch pollen ( $\mathrm{t} 3$ ) [9], and also in depth for timothy grass pollen (g6) [10]. In contrary to other rapid tests like the ImmunoCAP Rapid (Phadia, Uppsala, Sweden) [8], which employs a fixed panel of single allergens immobilized on membranes, ALFA utilizes liquid allergens and allergen mixtures. The open architecture of the ALFA system allows for the selection of the required allergen in combination with a universal basis set (see Figure 1).
Biotinylated insect venoms of honey bee, Apis mellifera (BV) and wasp, Vespula vulgaris and germanica (WV) were characterized and prepared for use with ALFA. The objective of this study was the evaluation of ALFA, for the detection of sIgE to bee venom (BV) and wasp venom (WV) in a well-defined cohort of patients. Sera were also analyzed by ImmunoCAP (Phadia, Uppsala, Sweden) and ALLERG-O-LIQ (Dr. Fooke Laboratorien $\mathrm{GmbH}$ ). The correlation between methods was calculated, and agreement between ALFA and the diagnosis (based on patient's history, positive skin test, and detection of sIgE by ImmunoCAP $(\geq 0.35 \mathrm{kU} / \mathrm{L}))$ was determined.

\section{Patients and Methods}

Sera of four groups were analyzed as follows.

(A) 40 patients with insect venom allergy to either bee $(n=12)$ or wasp $(n=28)$ venom with agreement of patient's history, sIgE against the given venom by ImmunoCAP and skin test. Mean age $41.8 \mathrm{y}$ (SD 14.96y). 23 (57.5\%) males, 17 (42.5\%) females. 


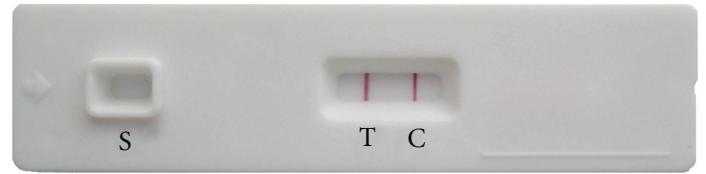

(a)

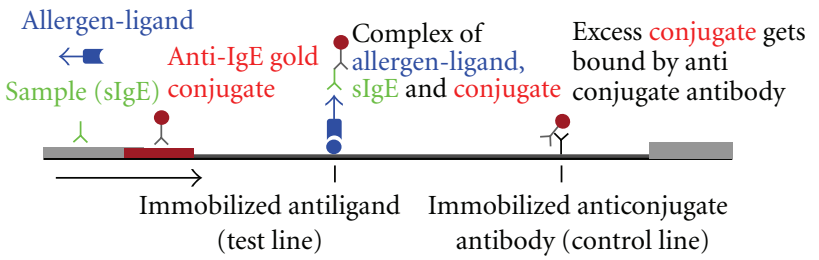

(b)

Figure 1: Principle of ALFA. A test cassette showing a positive test result is presented in (a) and the principle of the test in (b). The patient's sample is transferred to the sample application point. Immediately afterwards the allergen solution of interest is applied. During incubation time of $20 \mathrm{~min}$, the liquid is driven through the device by capillary flow. Allergen specific IgE of the sample binds specifically to the corresponding antigens of the allergen solution. The antigens are labeled and are retained at the test line $(\mathrm{T})$ by a capture molecule. At the same time the sIgE bound to the allergen is bound by an antibody coupled to colored particles (conjugate). The intensity of the color reaction at the test line is proportional to the amount of immune complexes consisting of ligand tagged antigens, sIgE, and IgE specific conjugate. The signal intensity ranges from faintly pink (low titer of sIgE) to dark ruby (high titer of sIgE). Access conjugate, which is not bound at the test line, will form a dark ruby control line (C) after 20 min of incubation.

$3(7.5 \%)$ Patients presented with anaphylactic reactions grade 1 (according to Ring and Messmer [11, $12]), 23(57.5 \%)$ with anaphylactic reaction grade 2 and $14(35 \%)$ with anaphylactic reaction grade 3 to insect venom sting. None of the patients presented with a grade 4 anaphylaxis. None of the patients were under immunosuppressive medication or specific immunotherapy. Mean total IgE was $78.7 \mathrm{kU} / \mathrm{L}$ (range 11.6-372 kU/L).

(B) 40 patients with insect venom allergy and double sensitization (serological) to bee and wasp venom, determined by ImmunoCAP, patient's history and skin test. Mean age 42.25 y (SD 17.5 y). 20 (50\%) males, $20(50 \%)$ females. 4 (10\%) Patients presented with anaphylactic reactions grade 1 (according to Ringb and Messmer $[11,12]), 27(67.5 \%)$ with anaphylactic reaction grade 2 and $9(22.5 \%)$ with anaphylactic reaction grade 3 to insect venom sting. None of the patients presented with a grade 4 anaphylaxis. None of the patients were under immunosuppressive medication or specific immunotherapy. Mean total $\operatorname{IgE}$ was $150.5 \mathrm{kU} / \mathrm{L}$ (range 16.5-667 kU/L).

(C) Atopic individuals ( $\mathrm{Sx} 1$ positive, total $\operatorname{IgE}$ mean = $2986 \mathrm{kU} / \mathrm{L}$, range $186-23813 \mathrm{kU} / \mathrm{L}$, history of at least one atopic disease) without history of insect venom allergy $(n=30)$. Mean age $33.5 \mathrm{y}(\mathrm{SD} 15.75 \mathrm{y})$.
16 (53.34\%) males, 14 (46.67\%) females. 19 (63.34\%) patients presented with atopic dermatitis, 12 (40\%) patients with allergic rhinoconjunctivitis, and 7 (23.34\%) with allergic asthma (some patients presented with two or three manifestations). None of the patients were under immunosuppressive medication or specific immunotherapy.

(D) Non atopic individuals without history of insect venom allergy $(n=30)$. None of the patients were under immunosuppressive medication or specific immunotherapy. Mean total $\operatorname{IgE}$ was $35.60 \mathrm{kU} / \mathrm{L}$ (range 2-99.3 kU/L).

Diagnosis of insect venom allergy was based on patient's history (anaphylaxis due to bee or wasp sting [11, 12]), positive skin test, and detection of sIgE by ImmunoCAP $(\geq 0.35 \mathrm{kU} / \mathrm{L})$.

2.1. Skin Test. In patients with insect venom allergy, skin prick tests with increasing concentrations of BV and WV (1, $10,100 \mu \mathrm{g} / \mathrm{mL}$ ) (ALK- Abello, Reinbeck, Germany) were performed. If negative, intradermal tests at $1 \mu \mathrm{g} / \mathrm{mL}$ were added as recommended by the position paper of the EAACI Interest Group on Insect Venom Hypersensitivity [13, 14]. Histamine chloride at a concentration of $1 \mathrm{mg} / \mathrm{mL}$ served as positive and saline solution $(0.9 \%)$ as negative control.

2.2. Detection of $\operatorname{IgE}$. All sera in this study $(n=140)$ were assayed for sIgE to BV and WV by ALFA, ALLERG-O-LIQ, a reversed-type, quantitative, WHO 75/502 calibrated immunoassay [1], and ImmunoCAP according to the instructions given by the manufacturer. Specific IgE values $>100 \mathrm{IU} / \mathrm{mL}$ were considered as $100 \mathrm{IU} / \mathrm{mL}$. ALFA results were quantified by a scanning device with an appropriate software [10], which measures the colour intensity of the test line and evaluates the validity of the test run by measuring the existence and intensity of the control line. All samples were measured after the same incubation time of $20 \mathrm{~min}$. The test values are converted into relative units (RUs). Using individually diluted allergen solutions, the test kits are validated for identical discrimination of positive and negative results. Cut-off values were set using ROC-decision analysis made with Analyze-it v2.21 for Excel (data not shown).

2.3. Statistical Analysis. Spearman correlation, P-values (1tailed Student's $t$-test), and Receiver Operating Characteristic analysis were performed with GraphPad Prism Ver. 5.01.

\section{Results and Discussion}

3.1. Detection of sIgE to Bee and Wasp Venom. When compared to the diagnosis (based on patients history, skin test, and SIgE detection by ImmunoCAP) comparative receiver operating characteristic analysis of groups A and B revealed area under the curve values for ALFA of 0.97 (BV) and 0.91 (WV).

In monosensitized patients (group A only), 12/12 BV allergic patients and $23 / 28 \mathrm{WV}$ allergic patients were positive in ALFA using a cut-off value for ALFA of 10.0 RU, 


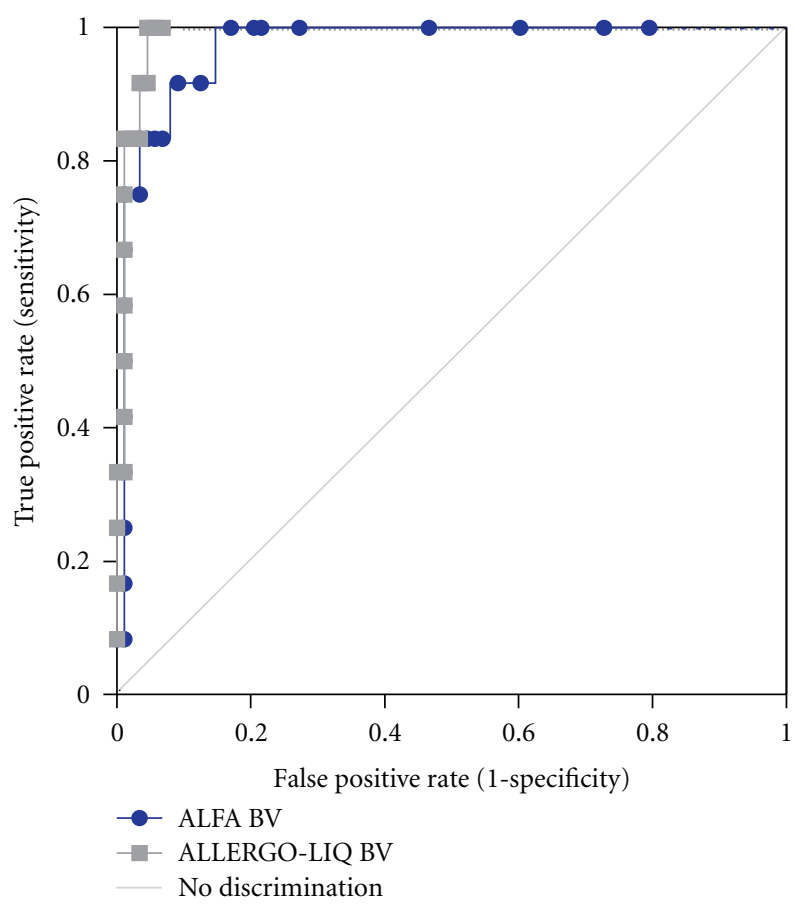

(a)

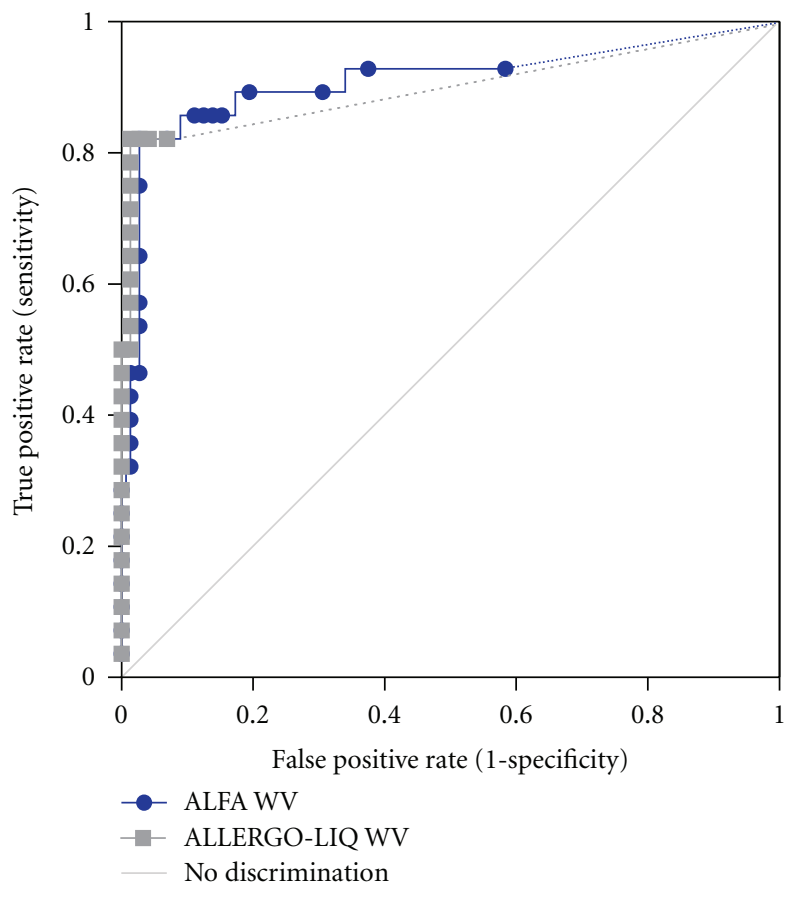

(b)

FIGURE 2: Receiver operating characteristic of ALFA and ALLERGO-LIQ for the diagnosis of bee (a) and wasp (b) venom allergy in monosensitized patients (group A) and control groups (C and D). Curve with dots indicates results for ALFA and curve with squares for ALLERGOLIQ. Diagnosis of insect venom allergy was based on patient's history, skin testing, and detection of sIgE to bee or wasp venom by ImmunoCAP. Sensitivity/specificity for ALFA is $100 \% / 83 \%(\mathrm{BV})$ and $82 \% / 97 \%$ (WV) at a cut-off value of $10.0 \mathrm{RU}$ and $100 \% / 93 \%$ (BV) and $82 \% / 93 \%$ (WV) for ALLERG-O-LIQ.

corresponding to a sensitivity of $100 \%$ for BV and $82 \%$ for WV. Similar results were obtained by ALLERG-O-LIQ. In ALLERG-O-LIQ 12/12 BV allergic patients and 24/28 WV allergic patients were found positive (Figure 2).

For groups $\mathrm{A}$ and $\mathrm{B}$ high agreement between ALFA, ALLERG-O-LIQ, and skin tests of up to $92 \%$ was observed (data not shown). Using atopic (without history of insect venom allergy - group C) and nonatopic (group D) sera as controls, the specificity of ALFA for the detection of sIgE to $\mathrm{BV}$ was calculated as $83 \%$ and for $\operatorname{sIgE}$ to $\mathrm{WV}$ as $97 \%$ (Figure 2).

3.2. Comparison of ALFA and ImmunoCAP. For insect venom allergic patients (groups A and B) the Spearman correlation coefficient for ALFA versus ImmunoCAP was 0.79 $(P<0.0001)$ for BV and $0.80(P<0.0001)$ for WV (data not shown). Correlation of ALFA to ImmunoCAP for each group revealed a more defined picture. For the detection of $\operatorname{sigE}$ to BV the Spearman correlation coefficient for ALFA versus ImmunoCAP was $0.72(P<0.0001)$ for group $\mathrm{A}, 0.86(P<$ 0.0001 ) for group $\mathrm{B}, 0.07$ (ns) for group $\mathrm{C}$, and 0.18 (ns) for group D (Figure 3). For the detection of sIgE to WV the Spearman correlation coefficient for ALFA versus ImmunoCAP was $0.82(P<0.0001)$ for group A, $0.84(P<0.0001)$ for group $\mathrm{B}, 0.25$ (ns) for group $\mathrm{C}$, and $0.31(P<0.05)$ for group D (Figure 4).

These results demonstrate a good quantitative agreement for the detection of sIgE to BV and WV between ALFA and
ImmunoCAP for sera of allergic patients (groups A and B) but poor quantitative agreement for the control groups, especially for BV (groups C and D). This seems to be caused by a surprisingly high number of positive ImmunoCAP results in the control groups, in particular in the group of atopic individuals (without history of insect venom allergy) with high total IgE-a finding that has been described before for different allergens $[15,16]$. In the present study in group C (atopic individuals with high total IgE) $19 / 30(63.3 \%)$ of the samples were BV positive by ImmunoCAP compared to 4/30 (13.3\%) samples tested positive by ALFA. For WV 9/30 (30.0\%) samples tested positive by ImmunoCAP and 1/30 sample (3.3\%) by ALFA. In group D positive BV results were observed for $3 / 30$ sera (10\%) by ImmunoCAP and $6 / 30$ (20.0\%) samples tested positive using ALFA. For WV the results in group D showed 4/30 (13.3\%) positive tests by ImmunoCAP and $1 / 30(3.3 \%)$ positive samples for ALFA, respectively, (Table 1). The different results of ALFA and ImmunoCAP in relation to total IgE level of the patient are shown in Figure 5.

Since sIgE to CCDs (cross-reactive carbohydrate determinants) can be responsible for major cross-reactivity of glycosylated allergens (e.g., of pollen, foods, insect venom, etc.), sIgE to CCDs could also account for the high rate of BV ImmunoCAP positives in the atopic negative controls (Group C). However analyzing CCD reactivity by ImmunoCAP showed that only less than $50 \%$ of the ImmunoCAP BV positive sera also displayed CCD reactivity (data not shown). 
TABLE 1: Results for the detection of bee and wasp venom of ALFA and ImmunoCAP for group A $(n=40)$, group B $(n=40)$, group C $(n=30)$, and group $\mathrm{D}(n=30)$.

\begin{tabular}{|c|c|c|c|c|c|c|c|c|}
\hline & \multirow[b]{2}{*}{$\begin{array}{l}\text { Bee venom } \\
\text { positive }\end{array}$} & \multicolumn{2}{|c|}{ ImmunoCAP } & \multicolumn{5}{|c|}{ ALFA (cut-off 10 RU) } \\
\hline & & $\begin{array}{c}\text { Bee venom } \\
\text { negative }\end{array}$ & $\begin{array}{c}\text { Wasp venom } \\
\text { positive }\end{array}$ & $\begin{array}{c}\text { Wasp venom } \\
\text { negative }\end{array}$ & $\begin{array}{c}\text { Bee venom } \\
\text { positive }\end{array}$ & $\begin{array}{c}\text { Bee venom } \\
\text { negative }\end{array}$ & $\begin{array}{c}\text { Wasp venom } \\
\text { positive }\end{array}$ & $\begin{array}{c}\text { Wasp venom } \\
\text { negative }\end{array}$ \\
\hline Group A & 12 & 28 & 28 & 12 & 17 & 23 & 23 & 17 \\
\hline Group B & 36 & 4 & 39 & 1 & 27 & 13 & 30 & 10 \\
\hline Group C & 19 & 11 & 9 & 21 & 4 & 26 & 1 & 29 \\
\hline Group D & 3 & 27 & 4 & 26 & 6 & 24 & 1 & 29 \\
\hline
\end{tabular}

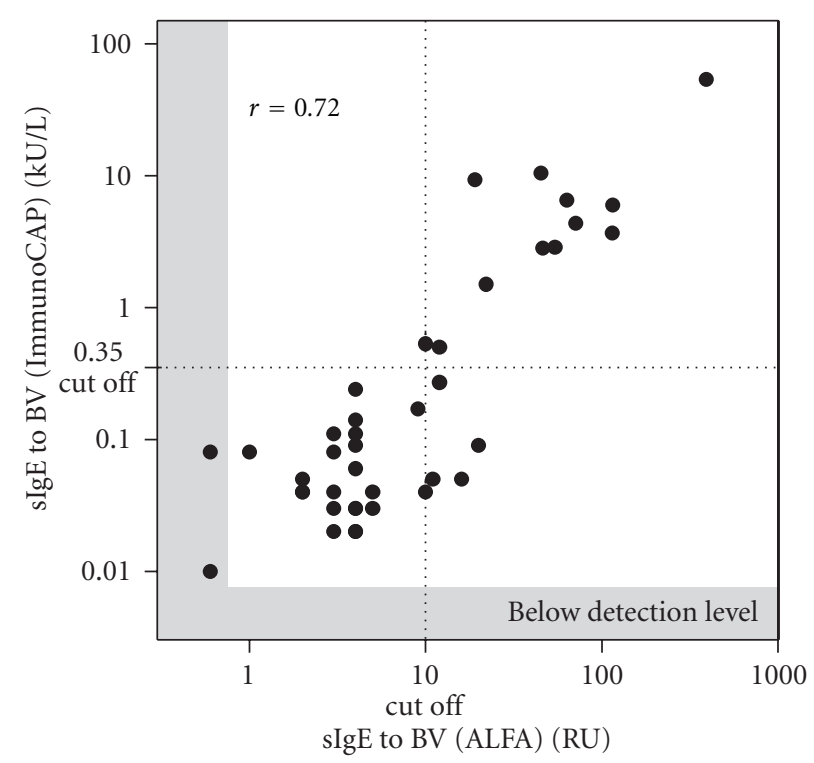

(a)

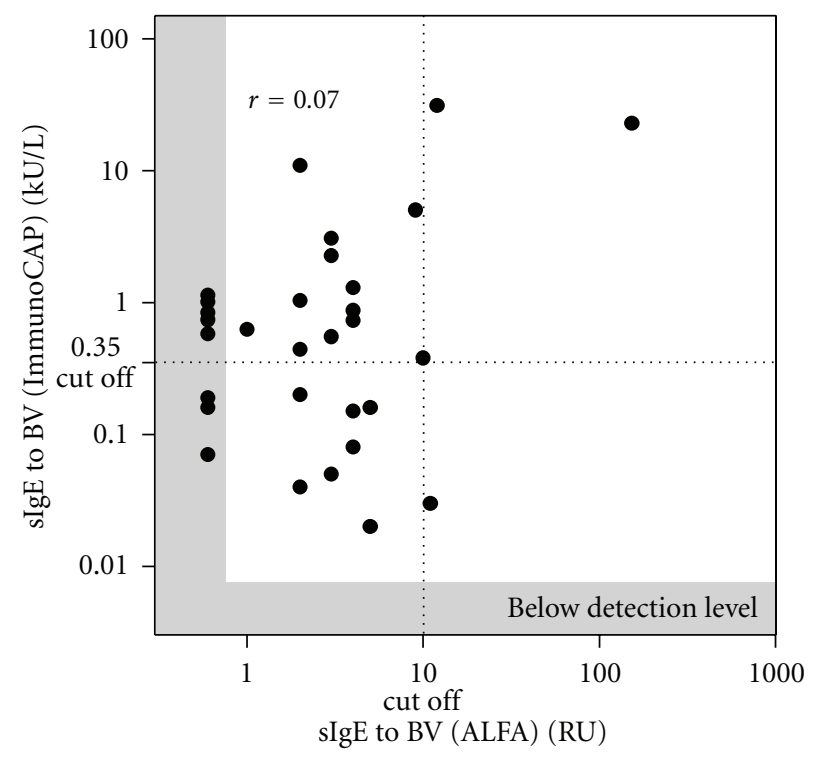

(c)

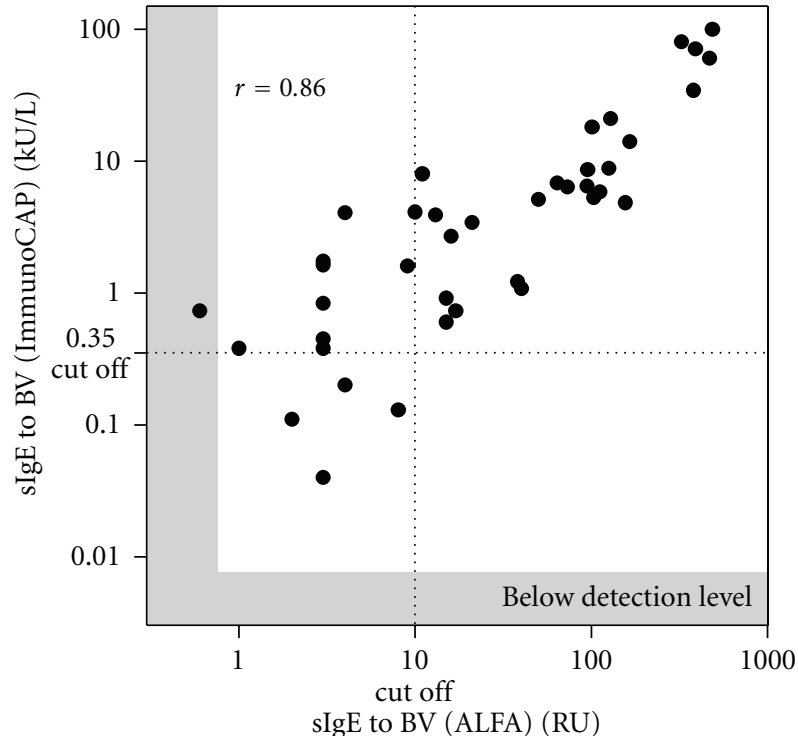

(b)

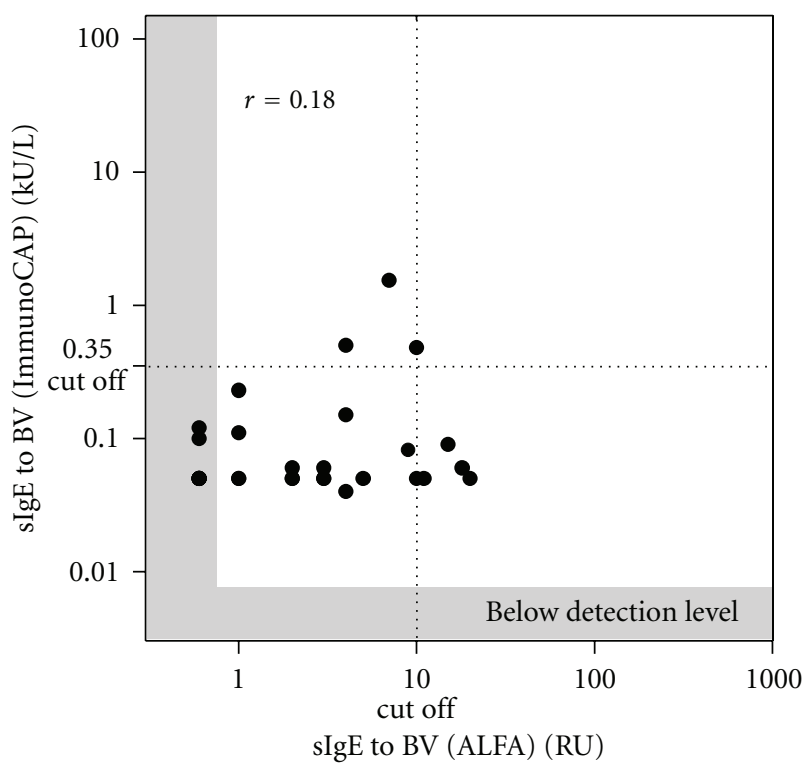

(d)

FIGURE 3: Spearman correlation diagram of ALFA versus ImmunoCAP for the detection of sIgE to bee venom. Quantitative agreement was found with a Spearman correlation coefficient of $0.72(P<0.0001)$ for group A (a), $0.86(P<0.0001)$ for group B (b), $0.07(P=0.37)$ for group C (c), and of $0.18(P=0.17)$ for group D $(d)$ for the detection of sIgE to BV. 


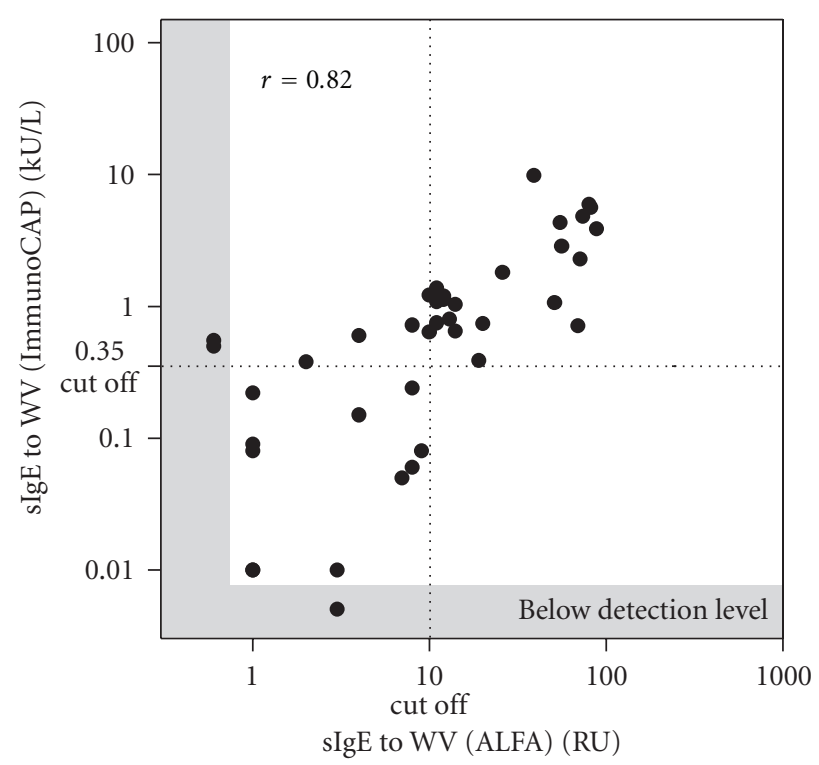

(a)

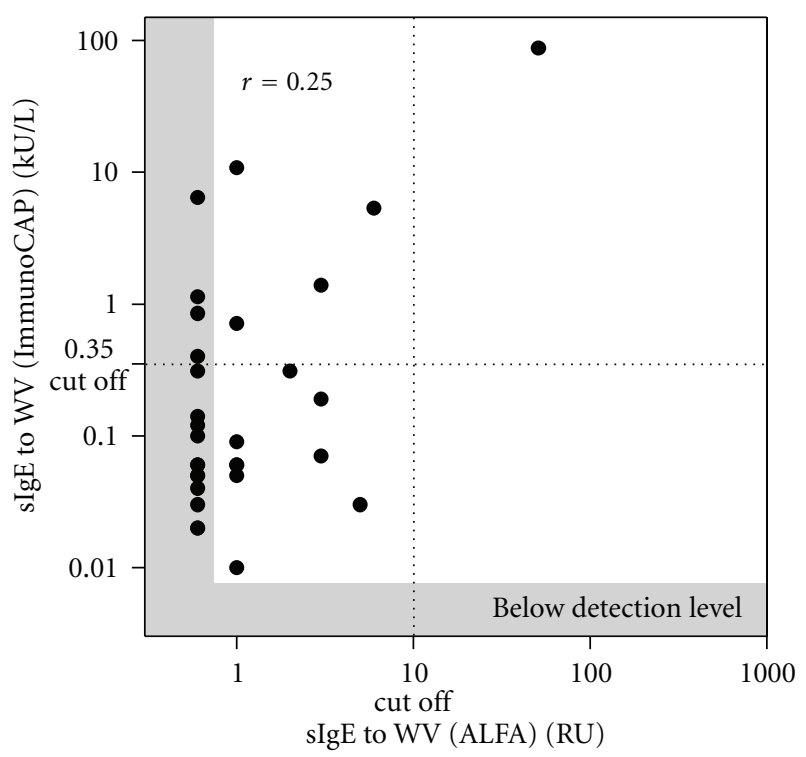

(c)

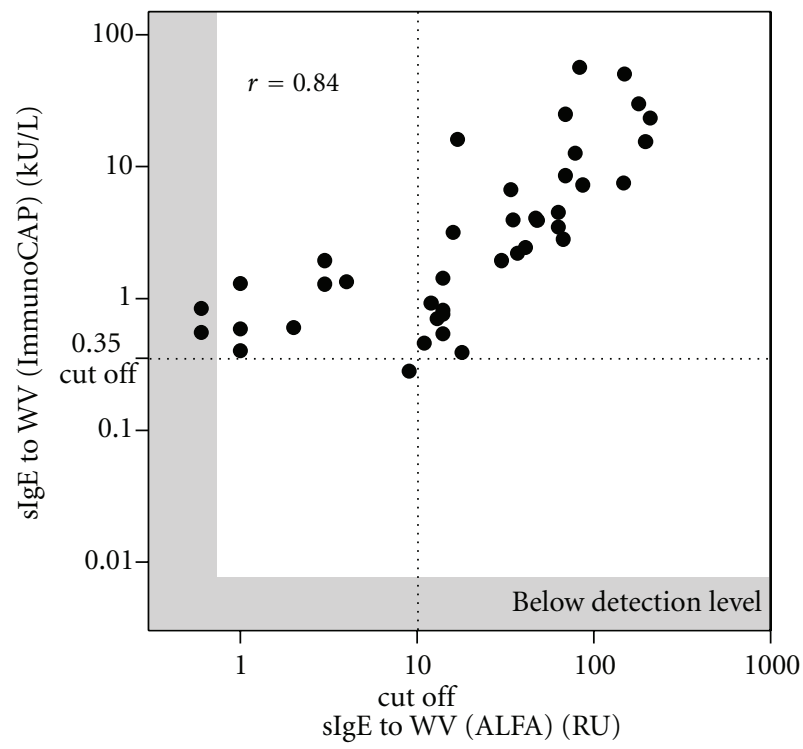

(b)

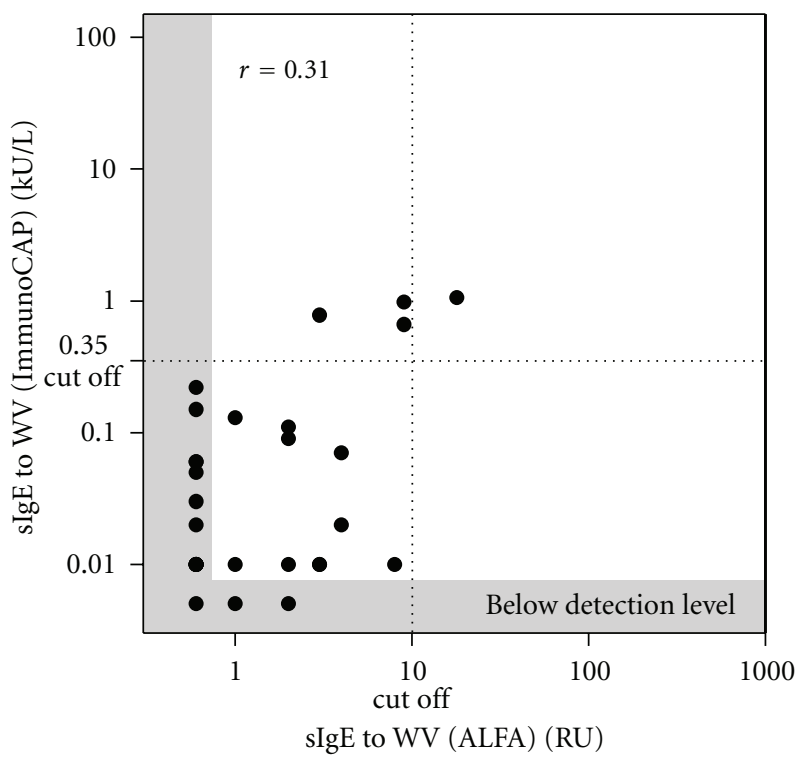

(d)

FIGURE 4: Spearman correlation diagram of ALFA versus ImmunoCAP for the detection of sIgE to wasp venom. Quantitative agreement was found with a Spearman correlation coefficient of $0.82(P<0.0001)$ for group A (a), $0.84(P<0.0001)$ for group B (b), $0.25(P=0.088)$ for group C (c), and of $0.31(P=0.047)$ for group D (d) for the detection of sIgE to WV.

This suggests that the high rate of BV positive results by ImmunoCAP in atopic patients (without history of insect venom allergy) with strongly elevated total IgE levels (mean $=2986 \mathrm{kU} / \mathrm{L}$, range $186-23813 \mathrm{kU} / \mathrm{L}$ ) cannot be explained by CCD reactivity but rather may reflect nonspecific IgE binding due to the solid phase architecture of the ImmunoCAP assay system.

Recently recombinant allergens have been introduced into the in vitro diagnostic of insect venom allergy. In particular for dissecting true double sensitization from crossreactivity recombinant allergens seems to be of great relevance [17-19]. Since ALFA's open architecture allows for the use of any biotinylated allergen, the introduction of recombinant bee and wasp venom allergens will be useful and will certainly further increase the clinical sensitivity and specificity.

\section{Conclusion}

Detection of sIgE to bee and wasp venom by ALFA shows in the present study a good discrimination between sera of bee or wasp allergic patients and control sera with a comparable performance to the ALLERG-O-LIQ and ImmunoCAP system. Noteworthy ALFA detected no sIgE to BV in WV 


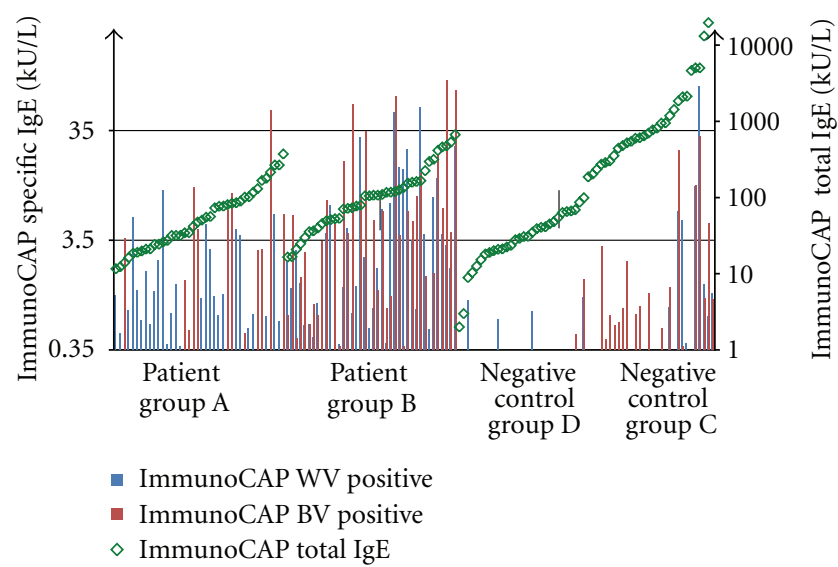

(a)

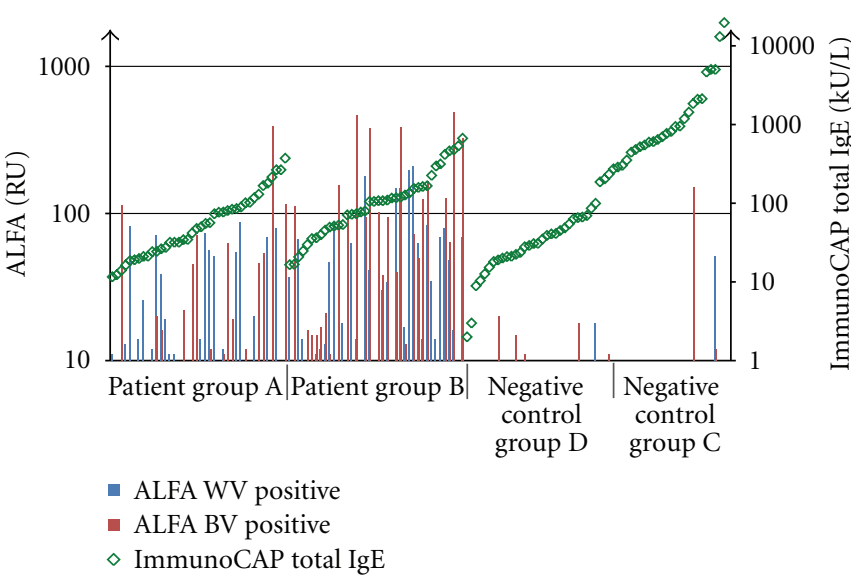

(b)

FIGURE 5: Graphical illustration of the relation of total IgE (green diamond, determined by ImmunoCAP) and sIgE to BV (red) and WV (blue) for ImmunoCAP (a) and ALFA (b) for each study group.

allergic patients and vice versa. In case of specificity, a high degree of correlation was found for ALFA and ALLERGO-LIQ, whereas differing results were obtained using the ImmunoCAP system.

We conclude that the Allergy Lateral Flow assay together with the novel scanner-based system represents a versatile and reliable tool for the measurement of sIgE to insect venom allergens meeting the growing demand for digital documentation of laboratory results. Further studies with more allergens (e.g., recombinant insect venom allergens) are mandatory to further proof the applicability of the ALFA test system.

\section{Conflict of Interests}

This study was supported in part by a research grant to T. Jakob by Dr. Fooke Laboratorien GmbH. N. Pfender declares that he has no conflict of interests. R. Lucassen, N. Offermann, J. Schulte-Pelkum, and M. Fooke are employees of Dr. Fooke Laboratorien GmbH.

\section{Authors' Contribution}

N. Pfender performed the experiments. N. Offermann, R. Lucassen, J. Schulte-Pelkum, and M. Fooke developed the test system. T. Jakob supervised the experiments. All authors have contributed equally to writing and corrections of the manuscript.

\section{Acknowledgment}

The authors thank Andrea Komann for excellent technical assistance.

\section{References}

[1] J. Kleine-Tebbe, K. Breuer, U. Lepp et al., "Spezifische IgEwerte gegen aero- und nahrungsmittelallergene im vergleich: Dr. Fooke ALLERG-O-LIQ und Pharmacia CAP-System," Allergologie, vol. 27, no. 4, pp. 129-135, 2004.
[2] R. G. Hamilton and N. Franklin Adkinson Jr., "In vitro assays for the diagnosis of IgE-mediated disorders," Journal of Allergy and Clinical Immunology, vol. 114, no. 2, pp. 213-226, 2004.

[3] W. K. Dolen, "IgE antibody in the serum-detection and diagnostic significance," Allergy, vol. 58, no. 8, pp. 717-723, 2003.

[4] S. G. Johansson, "Raised levels of a new immunoglobulin class (IgND) in asthma," The Lancet, vol. 2, no. 7523, pp. 951-953, 1967.

[5] R. G. Hamilton, "Diagnosis and treatment of allergy to hymenoptera venoms," Current Opinion in Allergy and Clinical Immunology, vol. 10, no. 4, pp. 323-329, 2010.

[6] U. Jappe, M. Raulf-Heimsoth, M. Hoffmann, G. Burow, C. Hübsch-Müller, and A. Enk, "In vitro hymenoptera venom allergy diagnosis: improved by screening for cross-reactive carbohydrate determinants and reciprocal inhibition," Allergy, vol. 61, no. 10, pp. 1220-1229, 2006.

[7] C. Diaz-Vazquez, M. J. Torregrosa-Bertet, I. Carvajal-Urueña et al., "Accuracy of immunoCAP rapid in the diagnosis of allergic sensitization in children between 1 and 14 years with recurrent wheezing: the IReNE study," Pediatric Allergy and Immunology, vol. 20, no. 6, pp. 601-609, 2009.

[8] P. A. Eigenmann, M. Kuenzli, V. D’Apuzzo et al., "The ImmunoCAP rapid Wheeze/Rhinitis child test is useful in the initial allergy diagnosis of children with respiratory symptoms," Pediatric Allergy and Immunology, vol. 20, no. 8, pp. 772-779, 2009.

[9] R. Lucassen, M. Fooke, J. Kleine-Tebbe, and M. Mahler, "Development and evaluation of a rapid assay for the diagnosis of immunoglobulin E-mediated type I allergies," Journal of Investigational Allergology and Clinical Immunology, vol. 18, no. 3, pp. 223-224, 2008.

[10] R. Lucassen, J. Schulte-Pelkum, C. Csuvarszki, J. Kleine-Tebbe, M. Fooke, and M. Mahler, "Evaluation of a novel rapid test system for the detection of allergic sensitization to timothy grass pollen against established laboratory methods and skin prick test," Journal of Allergy, vol. 2010, Article ID 524084, 4 pages, 2010.

[11] J. Ring and K. Messmer, "Incidence and severity of anaphylactoid reactions to colloid volume substitutes," The Lancet, vol. 1, no. 8009, pp. 466-469, 1977. 
[12] J. Ring, H. Behrendt, and A. De Weck, "History and classification of anaphylaxis," Chemical Immunology and Allergy, vol. 95, pp. 1-11, 2010.

[13] "Position paper: allergen standardization and skin tests. The European Academy of Allergology and Clinical Immunology," Allergy, vol. 48, pp. 48-82, 1993.

[14] B. M. Biló, F. Rueff, H. Mosbech et al., "Diagnosis of Hymenoptera venom allergy," Allergy, vol. 60, no. 11, pp. 1339-1349, 2005.

[15] M. Zidarn, M. Silar, M. Vegnuti, P. Korošec, and M. Košnik, "The specificity of tests for anti- $\beta$-lactam IgE antibodies declines progressively with increase of total serum IgE," Wiener Klinische Wochenschrift, vol. 121, no. 9-10, pp. 353-356, 2009.

[16] G. J. Sturm, A. Heinemann, C. Schuster et al., "Influence of total IgE levels on the severity of sting reactions in Hymenoptera venom allergy," Allergy, vol. 62, no. 8, pp. 884-889, 2007.

[17] S. C. Hofmann, N. Pfender, S. Weckesser, J. Huss-Marp, and T. Jakob, "Added value of IgE detection to rApi m 1 and rVes v 5 in patients with Hymenoptera venom allergy," Journal of Allergy and Clinical Immunology, vol. 127, no. 1, pp. 265-267, 2011.

[18] G. J. Sturm, W. Hemmer, T. Hawranek et al., "Detection of IgE to recombinant Api $\mathrm{m} 1$ and rVes $\mathrm{v} 5$ is valuable but not sufficient to distinguish bee from wasp venom allergy," Journal of Allergy and Clinical Immunology, vol. 128, no. 1, pp. 247-248, 2011.

[19] S. C. Hofmann, N. Pfender, S. Weckesser et al., "Detection of IgE to a panel of species specific allergens further improves discrimination of bee and wasp venom allergy," Journal of Allergy and Clinical Immunology, vol. 128, no. 1, 248 pages, 2011. 


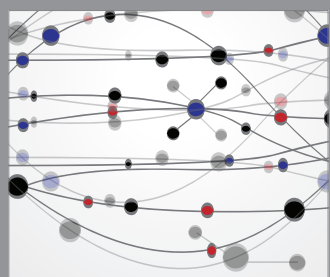

The Scientific World Journal
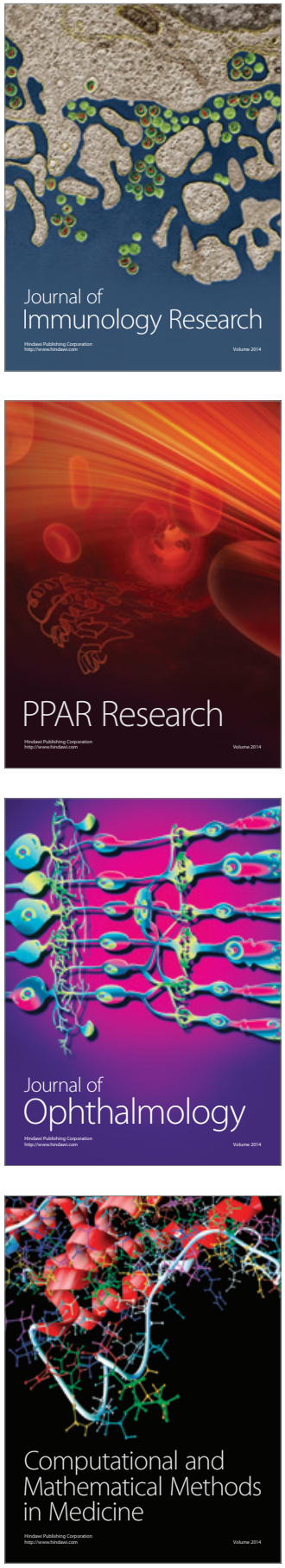

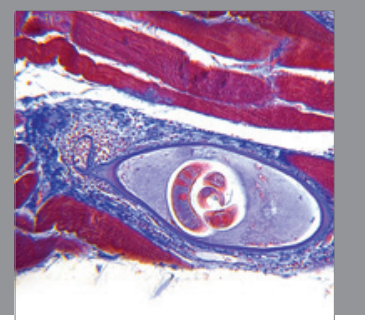

Gastroenterology

Research and Practice
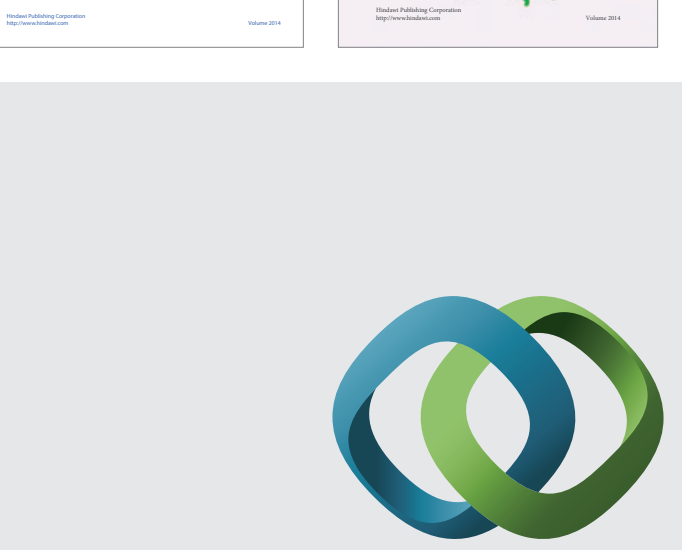

\section{Hindawi}

Submit your manuscripts at

http://www.hindawi.com
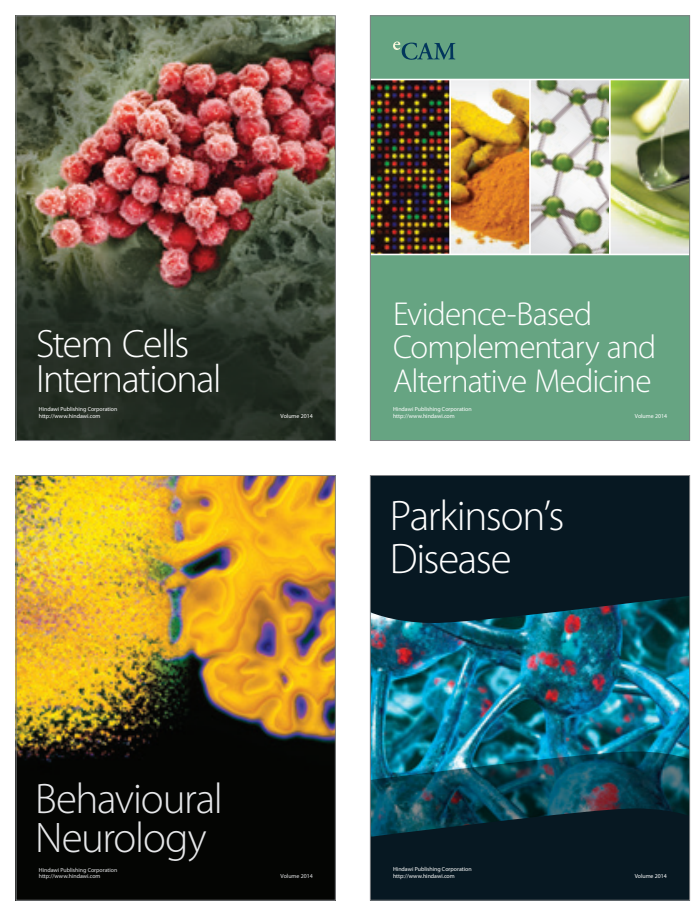

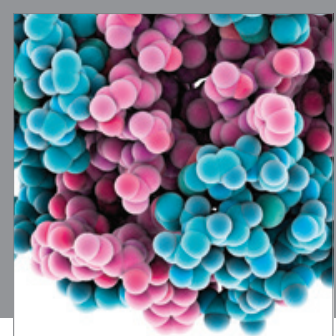

Journal of
Diabetes Research

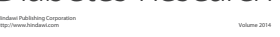

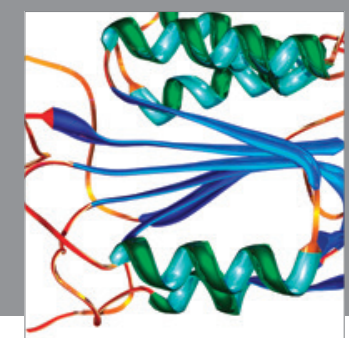

Disease Markers
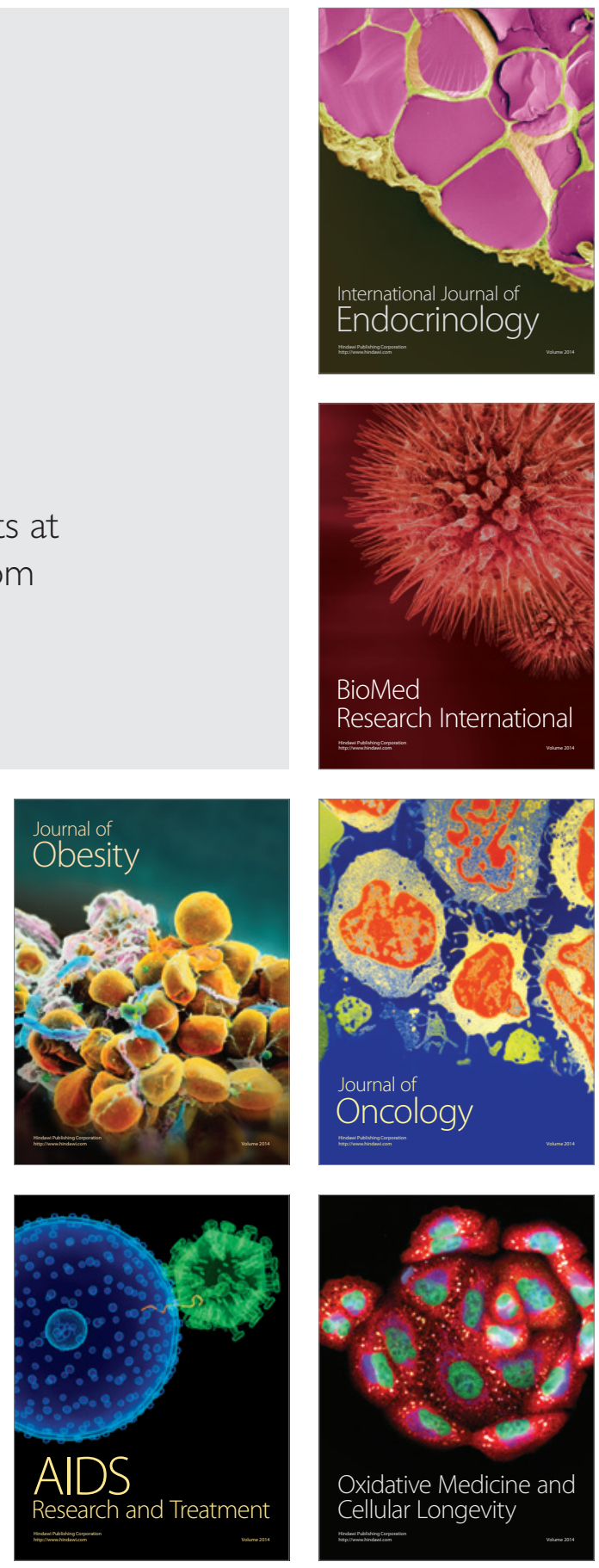